Published in final edited form as:

Sex Transm Dis. 2018 May ; 45(5): 294-300. doi:10.1097/OLQ.0000000000000739.

\title{
OPTIMIZING SCREENING FOR SEXUALLY TRANSMITTED INFECTIONS IN MEN USING SELF-COLLECTED SWABS - A SYSTEMATIC REVIEW
}

\author{
Nicholas Yared, MD*, Keith Horvath, $\mathrm{PhD}^{\dagger}$, Oluwaseun Fashanu, MBBS ${ }^{\dagger}$, Ran Zhao, BS ${ }^{\dagger}$, \\ Jason Baker, MD MS ${ }^{\star}, \neq$, and Shalini Kulasingam, $\mathrm{PhD}^{\dagger}$ \\ "Division of Infectious Diseases and International Medicine, Department of Medicine, University of \\ Minnesota, Minneapolis, MN \\ tDivision of Epidemiology and Community Health, School of Public Health, University of \\ Minnesota, Minneapolis, MN \\ ¥Minnesota Medical Research Foundation, Minneapolis, MN
}

\begin{abstract}
Background-Sexually transmitted infection (STI) rates are increasing in the U.S. while funding for prevention and treatment programs has declined. Sample self-collection for STI testing in men may provide an acceptable, easy, rapid and potentially cost-effective method for increasing diagnosis and treatment of STIs.
\end{abstract}

Methods-We conducted a systematic review of articles assessing self-collection of anal, oral, or genital swab samples among adult men for detection of STIs and/or human papillomavirus (HPV)related dysplasia. We searched for English-language articles in which men aged $\geq 18$ years were recruited to participate.

Results-Our literature search resulted in 1053 citations, with 20 meeting inclusion criteria. Self-collection methods were highly sensitive and comparable to clinician-collection for detection of multiple STI pathogens. However, self-collected samples were less likely to be of adequate quality for anorectal cytology and less sensitive for detection of anal intraepithelial neoplasia (AIN) than clinician-collected samples. Self-collection was highly acceptable. Overall, studies were small, heterogeneous, and used designs providing lower levels of evidence.

Conclusion-Self-collection methods are a viable option for collecting samples for STI testing in adult men based on their high feasibility, acceptability, and validity. Implementation of selfcollection procedures in STI testing venues should be performed to expand opportunities for STI detection and treatment.

Correspondence: Yared Nicholas MD, Division of Infectious Diseases and International Medicine, Department of Medicine, University of Minnesota, 420 Delaware Street SE, MMC 250, Minneapolis, MN 55455, USA. Phone Number: (612) 624-9996, Fax Number: (612) 625-4410, yared007@umn.edu.

Conflict of Interest: None declared 


\section{INTRODUCTION}

Reportable cases of sexually transmitted infections (STIs) have been rising in the United States with an increase in the incidence of the three most common STIs - chlamydia, gonorrhea, and syphilis - between 2014 and $2015 .{ }^{1}$ At the same time that STI transmission has been increasing, funding for STI prevention and treatment programs has been declining. This has resulted in a reduction in the number of clinics involved in STI diagnosis and treatment. ${ }^{1}$ Methods of specimen collection that are easy with a rapid turnaround time and low cost are needed to scale up identification and treatment of STIs.

Self-collection of biological specimens for STI testing offers potential screening benefits. These include greater participant ease with the collection process and the possibility of sample collection outside of traditional clinical settings. A meta-analysis comparing women performing home-based, self-collected sample acquisition versus women undergoing clinicbased sampling for STI screening showed greater rates of screening in the home-based collection group. ${ }^{2}$ Several studies involving women obtaining self-collected vaginal swabs have shown high acceptability of this collection method and validity of detection of chlamydia using polymerase chain reaction (PCR) amplication. . $^{3,4,5,6}$

Most of the initial studies involving self-collection focused on women as participants; however, evaluation of self-collection for STI screening for men would be useful since they are also adversely affected by a high burden of STIs. In particular, younger men and men who have sex with men (MSM) are at an elevated risk for STIs. Among men aged 20-24 years, rates of chlamydia and gonorrhea increased by $12.2 \%$ and $22.9 \%$, respectively, between 2011 and $2015 .{ }^{7}$ The rise of resistant strains of gonorrhea have also been of special concern in MSM with estimated incidence of gonorrhea among this group increasing from 1169.7 cases per 100,000 MSM in 2010 to 1474.4 cases per 100,000 MSM in $2013 .{ }^{8}$ Incidence rates of human papillomavirus (HPV)-related anal cancer, a long-term sequela of HPV infection, have increased among HIV-positive MSM despite wide-scale implementation of antiretroviral therapy use within the United States. ${ }^{9}$

We conducted a systematic review of studies of self-collected biological samples to better understand their potential utility for detection of STIs in adult men. Our main goals were to understand the use of self-collected samples for STI testing in men (of all sexual identities), the adequacy of self-collected samples, the validity of self-collected samples compared with clinician-collected samples, and the acceptability of the self-collection process.

\section{METHODS}

Preferred reporting items for systematic reviews and meta-analysis (PRISMA) guidelines were followed for this literature review. ${ }^{10}$ Identification and abstraction of articles involved the use of 4 electronic bibliographic databases: MEDLINE, EMBASE, CINAHL, and the Cochrane Central Register of Controlled Trials. 


\section{Eligibility Criteria}

Articles referenced for this study included abstracts and full-text articles published in peerreviewed journals. Review articles and editorials were omitted from the analysis. Articles were included based on presentation of original research whose methodology involved employment of self-collection techniques to obtain samples for testing for sexually transmitted infections (STIs) in men aged 18 and older. We focused specifically on selfcollection involving swabs of oral, genital, and/or anal sites. Studies that only had men selfcollect urine samples were excluded from this analysis.

\section{Search Strategy}

Databases were queried from January 2000 to August 2017. Both standardized medical subject heading (MeSH) terms and free text terms were used to identify articles of interest in all databases. A list of search terms was determined in advance for common usage among all electronic databases investigated. Iterations of "self-collect," "screen," "sexually transmitted diseases," and "sexually transmitted infections" were searched. Additionally, specific MeSH terms, including "Self Care," "Mass Screening," "Sexually Transmitted Diseases," and "Sexual Behavior" were searched in databases employing MeSH terms. Equivalent phrases were searched in EMBASE since this database uses its own Emtree terms. Where feasible, additional restrictions were used to include articles published only in English that included male participants aged 18 and older.

\section{Data Collection}

All abstracts generated by individual searches were screened to determine whether they merited eligibility screening for study inclusion. Full-text versions of articles meeting study inclusion criteria based on their abstracts were then obtained. The full-text articles underwent assessment of their eligibility to be included in the qualitative analysis by three reviewers as described in the next section. References not meeting inclusion criteria were quantified to calculate the number of articles excluded from analysis. Articles eligible for inclusion in the qualitative analysis were then recorded in a spreadsheet that consisted of the journal reference, authors, database searched, and search terms used to identify the article.

\section{Extraction and Eligibility Process}

An online eligibility form was created using Qualtrics Survey Software (Qualtrics, Provo, UT) to assess whether studies were eligible for inclusion in the systematic review. Information about the study design, geographic location of recruitment, intervention, and primary outcome were queried for each study reviewed. Information about the sex of participants involved in self-collection, whether self-collection results were specified for MSM or HIV-positive men, the types of swabs used for self-collection, and the microorganisms detected were also asked about in the eligibility form. Finally, each article was assessed for feasability and acceptability measures related to self-collection.

Three coders independently reviewed the full text articles for eligibility. Articles were initially double-coded with article assignment randomized to each coder. Discrepancies in data abstraction for all articles were then resolved by a third coder. 


\section{RESULTS}

A PRISMA flow chart showing the process of article identification, screening, and eligibility is presented in Figure 1. Of the 1053 citations initially identified, 20 studies were found to be eligible for inclusion in the review. Of the 20 studies included for analysis, most were cross-sectional $(\mathrm{n}=16)$ with a few other study types represented, including a cohort study ( $\mathrm{n}$ $=1)$, a qualitative study $(\mathrm{n}=1)$, and a quasi-experimental pretest-posttest study $(\mathrm{n}=1)$. One randomized trial was identified. ${ }^{24}$ Eight studies involved the use of self-collection of swabs for HPV detection. Additional micro-organisms for which self-collected swabs were obtained for STI testing included Chlamydia trachomatis $(\mathrm{n}=11)$, Neisseria gonorrhoeae ( $\mathrm{n}$ $=11)$, Trichomonas vaginalis $(\mathrm{n}=5)$, and Mycoplasma genitalium $(\mathrm{n}=2)$. One study evaluating the acceptability of self-sampling for oropharyngeal and rectal specimens did not present data about specific micro-organisms for which testing was performed. ${ }^{15}$

\section{HPV Testing}

Table 1 lists studies for which HPV prevalence was measured for matched self-collected and clinician-collected samples. ${ }^{11,12,24}$ Overall, self-collected samples showed comparable detection of HPV types to clinician-collected samples. Two studies performed by Hernandez et al. showed similar estimates of detection of all HPV types for participants collecting genital swabs using self-collected and clinician-collected methods. ${ }^{11,12} \mathrm{~A}$ study by Lampinen et al. showed similar estimates of detection of high-risk HPV DNA types using matched rectal swab samples with $67 \%$ of self-collected swabs and $62 \%$ of cliniciancollected swabs found to be positive. ${ }^{11}$

Two studies evaluated whether self-collected swabs submitted for anal cytology were of sufficient quality to be able to generate a cytology result. One study involving collection of swabs for anal cytology did not have a clinician-collected comparison group but did find that only $62.3 \%$ of self-collected anal swabs yielded samples of sufficient quality for interpretation of cytology results by a pathologist. ${ }^{18}$ In contrast, a study performed by Lampinen et al. in 2006 reported a higher overall proportion of self-collected swabs that were of sufficient quality to yield a cytology result, but was still less than that reported for paired clinician-collected swabs (83\% vs. $92 \%$, respectively, McNemar's chi-square with P <0.001). ${ }^{13}$ Unlike their ability to result in samples comparable to clinician-collected specimens for detecting HPV DNA, self-collected samples were less likely to meet the quality standards needed for interpretation of cytology to occur.

Chin-Hong et al. was the only study identified that compared the sensitivity and specificity of anal cytology, assessed via self- and clinician-collected anal swabs, to biopsy-proven anal intraepithelial neoplasia (AIN) in MSM. ${ }^{14}$ Study results were reported separately for HIVpositive and HIV-negative MSM. Specificity of abnormal anal cytology did not significantly differ between self-collected and clinician-collected specimens for HIV-positive MSM (50\% vs. $64 \%$ ) or HIV-negative MSM ( $86 \%$ vs. $85 \%$ ). However, self-collected samples were found to be less sensitive than clinician-collected samples for detection of AIN for both HIV-positive (75\% vs 90\%) and HIV-negative (48\% vs 62\%) MSM. ${ }^{13}$ 


\section{Chlamydia and Gonorrhea Testing}

Other sexually transmitted pathogens amenable to detection via the use of self-collected swabs in men include Chlamydia trachomatis and Neisseria gonorrhoeae. The introduction of self-collected swab techniques into clinics has been particularly favorable for detection of chlamydia and gonorrhea at extra-genital sites in particular subgroups of men (e.g. MSM). 16,29 One study using a pretest posttest design found that combined rates of detection in MSM of extra-genital chlamydia and gonorrhea increased by a factor of four following the introduction of self-collected swabs. ${ }^{29}$

A significant proportion of the literature related to self-collection for chlamydia and gonorrhea testing in men has focused on comparisons of self-collection techniques using different biological samples. Four studies compared self-collected swabs of the lower male genital tract (meatal, penile-meatal, or urethral swabs) to self-collected urine samples for detection of $C$. trachomatis and $N$. gonorrhoeae. ${ }^{21,22,23,30}$ Chernesky et al. found detection of both chlamydia (35 vs. 33 ) and gonorrhea (14 vs. 11) infection was slightly greater using meatal swab samples compared with urine samples, respectively, in a supervised clinic setting. ${ }^{21}$ This finding was replicated in a subsequent study by Chernesky et al. in which the rate of detection of $C$. trachomatis was found to be statistically significantly higher with the use of meatal swabs compared to the use of urine samples. ${ }^{30}$

A screening study conducted by Chai et al. in which men accessed test kits through the internet and mailed back home-collected urethral swabs and urine samples showed that the sensitivity of detection of $C$. trachomatis was higher for the penile swab samples (93\%) versus the urine samples (78\%). ${ }^{22}$ Complementing this data was a study that showed greater sensitivity of home-based self-collection of penile-meatal swabs for detection of chlamydia ( $94.2 \%$ sensitivity) and gonorrhea (100\% sensitivity), when compared to the use of urine samples $\left(76.7 \%\right.$ and $88.8 \%$ sensitivities, respectively). ${ }^{23}$

\section{Trichomoniasis and Mycoplasma genitalium Testing}

Unlike chlamydia and gonorrhea, trichomoniasis has only recently emerged as a condition for which testing in men has become feasible with the use of nucleic acid amplification assays (NAATs). Five studies evaluated the use of self-collected samples for testing for Trichomonas vaginalis infection in men. ${ }^{22,23,25,28,30}$ A study conducted by Chai et al. showed sensitivity of detection of $T$. vaginalis to be $82 \%$ with urethral swab collection versus only $40 \%$ with urine collection. ${ }^{22}$ Two studies evaluated the use of swabs involving collection at the penile meatus compared to urine collection, and both of them demonstrated higher rates of detection of $\mathrm{T}$. vaginalis via the use of meatal swabs. ${ }^{23,30}$ Of particular note was a study conducted by Dize et al. that showed a large difference in sensitivities for $T$. vaginalis detection between the use of self-collected penile-meatal swabs ( $80.4 \%$ sensitivity) and urine samples (39.3\% sensitivity). ${ }^{23}$ A subsequent study performed by Dize et al. noted a similar rate of sensitivity of $T$. vaginalis detection $(85 \%)$ when using clinician-collected urethral swabs as a gold standard for collection. ${ }^{28}$ Finally, one study examining selfcollected samples for evaluation of trichomoniasis in men did not include a comparative sample collection method for description in this context. ${ }^{25}$ Overall, quite large differences in 
sensitivity for detecting $T$. vaginalis emerged among studies where self-collected swabs were compared against self-collected urine samples.

Mycoplasma genitalium has been linked to the development of urethritis in men although diagnostic testing for this pathogen is limited to research-use-only (RUO) NAATs found in a few centers globally. ${ }^{31}$ Nevertheless, obtaining self-collected swabs for the detection of $M$. genitalium in men is a current area of exploration given the growing interest in the potential pathogenicity of this organism. One study using a transcription-mediated Amp based RUO test noted greater detection of $M$. genitalium with the use of meatal swabs (15.3\%) compared to first catch urine samples $(12.6 \%) .{ }^{30}$ While that study looked favorable for the ability of meatal swabs to detect $M$. genitalium, a study performed by Dize et al. using penile-meatal swabs noted a detection sensitivity of only $79.3 \% .{ }^{28}$ Of note, this sensitivity calculation used the assumption that clinician-collected urethral swabs were the gold standard for detection of M. genitalium.

\section{Feasibility and Acceptability of Swab Self-Collection}

Four studies focused on the feasibility of performing self-collection in settings outside of traditional clinics. ${ }^{18,22,27,28}$ One study evaluated the prevalence of HPV infection in MSM by recruiting men from venues that included bars, bathhouses, bookstores, community organizations, and community events that targeted gay and bisexual men. Two hundred and sixty eight individuals in this study self-collected rectal swabs; of the 498 who were approached and opted not to participate, $59.1 \%$ declined because of discomfort with the selfcollection method while $11.4 \%$ cited discomfort with engaging in self-collection at one of the aforementioned venues. ${ }^{18}$ In contrast, the study by Wood et al. found that $100 \%$ of the first thirty participants approached to participate in a nurse-delivered outreach screening service for self-collected STI testing at a sauna agreed to the use of self-collection for chlamydia and gonorrhea screening. ${ }^{27}$ Regarding ease of self-collection, a majority of participants in all studies where this aspect of feasibility was measured rated self-collected swabs as "very easy" or "easy" to use. ${ }^{16,22,28}$

Several studies examined the acceptability of the self-collection process. One study evaluated acceptability in terms of participants' willingness to perform self-collection; it found that of a subset of HIV-positive MSM, 92\% opted to perform self-collected rectal swabs as part of a sexual health screen. ${ }^{26}$ Acceptability was also measured in terms of participant satisfaction with the self-collection process. Using this definition, one study found that $76 \%$ of men self-collecting pharyngeal swabs and $82 \%$ of men self-collecting rectal swabs found the self-collection process to be acceptable. ${ }^{15}$ Another study found that > $90 \%$ of surveyed participants who had undergone self-collection of rectal swabs would undergo self-collection again and would return to an STI clinic if self-collection were the standard method of testing for rectal STIs. ${ }^{16}$ Finally, a study involving the use of a nurse-led self-screening program for detection of $C$. trachomatis and $N$. gonorrhoeae found that all twenty-one MSM completing a patient satisfaction questionnaire about self-collected pharyngeal and rectal swabs after having obtained these samples noted self-collection to be an acceptable screening method. ${ }^{19}$ 
Two studies in the review examined participants' perceptions of the use of different types of swabs for self-collection. ${ }^{21,24}$ Chernesky et al. found that male participants thought firstcatch urine sampling was easier and more comfortable than self-collection of swabs for chlamydia and gonorrhea screening. ${ }^{20}$ Hernandez et al. compared two different types of dry swabs and a wet swab method for detection of HPV from male penile-meatal samples.

Swabs were rubbed over the entire surface of the penis from the tip of the glans to the base of the penile shaft with the wet swab method involving application of emery paper prior to application of a saline-moistened swab. The study found greater comfort, less pain, and less time required for swab collection among participants using the dry swab methods compared to the wet swab method. ${ }^{24}$

\section{DISCUSSION}

We identified twenty studies in which self-collected swab samples were obtained from adult men with the goal to submit these for STI testing. Our review demonstrates that the majority of studies used a cross-sectional design with small sample sizes, indicative of the relatively new emergence of this collection method for use in men. Many studies were identified that incorporated self-collection of samples into their methods but only briefly analyzed the selfcollection process itself or evaluated it as a secondary outcome. For example, nine studies that would have met criteria for inclusion in the review were excluded because no STI outcome measures from self-collected samples were examined.

Self-collected samples were generally of sufficient quality for detection of specific HPV types in comparison to clinician-collected samples. However, the ability to yield swab samples that were of sufficient quality to generate a cytology result was less for selfcollected samples compared to clinician-collected samples. ${ }^{13,17}$ Conducting larger studies comparing clinician-collection to different self-collection practices and evaluating different methods of communicating the self-collection process to participants would be useful to further investigate whether self-collection is a reliable method for generating samples of sufficient quality to undergo cytology testing.

Self-collection of samples was found to perform as well as or better than clinician-collection for STI detection in several studies detailed in this review. ${ }^{11,12,21,23,28}$ Given concerns about underdiagnosis of STIs, self-collection has the potential to complement and expand upon current screening efforts, in part, because of greater comfort and control of the collection process, patient privacy, and the ability to collect samples at home or in other non-clinical venues. A high level of willingness to undergo self-collection was seen among male participants in several studies, consistent with findings noted for performance of selfcollection in prior studies involving women. ${ }^{15,16,19,26}$ However, conflicting perceptions about the use of swabs for self-collection was observed, as evident by men's preference for collecting urine samples over swab samples in one study and the preference for use of dry swabs over wet swabs in another. ${ }^{20,24}$ More studies are needed to determine which attributes of different self-collection methods are associated with increased feasability and acceptability given the potential impact of self-collection on designing successful interventions to promote STI screening. 
Our systematic review has several limitations. Our search strategy focused on Englishlanguage articles and articles featuring adults. Most of the studies evaluating self-collection methods consisted of cross-sectional studies with only one randomized trial identified. This limitation affects the sensitivity and specificity estimates of self-collection versus cliniciancollection. Confounding may also affect the validity of any comparisons although the use of paired self- and clinician-collected swabs in some studies did help to partially counteract this. Cross-sectional studies that analyzed the acceptability of self-collection may be prone to bias since participants who entered these studies may have been more inclined to support or accept self-collection methods. However, the consistency of high acceptability rates seen for self-collection methods across studies is consistent with literature showing similar findings for other groups and helps to bolster the notion that self-collection methods are indeed acceptable for men.

Another important limitation of this review was the heterogeneity among studies that limited our ability to pool data across studies for a meta-analysis. Sources of heterogeneity included the type of swab used, the anatomic site at which self-collection was performed, and the pathogenic micro-organism targeted for detection. This heterogeneity is likely a reflection of the continued novelty of performing self-collection for STI testing.

Despite these limitations, self-collection of swab samples in men has the potential to be a viable mode of STI detection given its comparable sensitivity for pathogen detection to clinician-collected samples and its high acceptability. Studies involving larger numbers of participants with robust randomization schemes, continued innovation in swab design, and communication of self-collection instructions would help to inform future protocols for healthcare and community organizations interested in improving STI detection and treatment rates. Innovative strategies to curb and reduce the growing burden of STIs in men remain important, and self-collection has the potential to play a significant role in achieving the desired improvements.

\section{Acknowledgments}

Sources of Support: Dr. Nicholas Yared was funded by the National Institutes of Health Infectious Disease Training in Clinical Investigation Grant (T32 AI055433-11A1).

\section{References}

1. National Center for HIV/AIDS, Viral Hepatitis, STD, and TB Prevention. Reported STDs at Unprecedented High in the U.S. [Internet]. Atlanta (GA): Centers for Disease Control and Prevention; 2016 Oct 19. Available at: http://www.cdc.gov/nchhstp/newsroom/2016/stdsurveillance-report-2015-press-release.html [Accessed 2017 Feb 9]

2. Odesanmi TY, Wasti SP, Odesanmi OS, et al. Comparative Effectiveness and Acceptability of Home-Based and Clinic-Based Sampling Methods for Sexually Transmissible Infections Screening in Females Aged 14-50 Years: A Systematic Review and Meta-Analysis. Sex Health. 2013; 10(6): 559-569. [PubMed: 24160747]

3. Holland-Hall CM, Wiesenfeld HC, et al. Self-collected Vaginal Swabs for the Detection of Multiple Sexually Transmitted Infections in Adolescent Girls. J Pediatr Adolesc Gynecol. 2002; 15(5):307313. [PubMed: 12547662] 
4. Wiesenfeld HC, Lowry DL, Heine RP, et al. Self-collection of Vaginal Swabs for the Detection of Chlamydia, Gonorrhea, and Trichomoniasis: Opportunity to Encourage Sexually Transmitted Disease Testing Among Adolescents. Sex Transm Dis. 2001; 28(6):321-325. [PubMed: 11403188]

5. Newman SB, Nelson MB, Gaydos CA, Friedman HB. Female Prisoners' Preferences of Collection Methods for Testing for Chlamydia trachomatis and Neisseria gonorrhoeae Infection. Sex Transm Dis. 2003; 30(4):306-309. [PubMed: 12671549]

6. Chernesky MA, Hook EW 3rd, Martin DH, et al. Women Find It Easy and Prefer to Collect Their Own Vaginal Swabs to Diagnose Chlamydia trachomatis or Neisseria gonorrhoeae Infections. Sex Transm Dis. 2005; 32(12):729-733. [PubMed: 16314768]

7. National Center for HIV/AIDS, Viral Hepatitis, STD, and TB Prevention. Sexually Transmitted Disease Surveillance 2015. [Internet]. Atlanta (GA): Centers for Disease Control and Prevention; 2016 Oct. Available at: https://www.cdc.gov/std/stats15/default.htm [Accessed 2017 Feb 27]

8. National Center for HIV/AIDS, Viral Hepatitis, STD, and TB Prevention. STDs in Men Who Have Sex with Men. [Internet]. Atlanta(GA): Centers for Disease Control and Prevention; 2016 Oct 18. Available at: https://www.cdc.gov/std/stats15/msm.htm [Accessed 2017 Feb 9]

9. Crum-Cianflone NF, Hullsiek KH, Marconi VC, et al. Anal Cancers among HIV-Infected Persons: HAART is Not Slowing Rising Incidence. AIDS. 2010; 24(4):535-543. [PubMed: 19926961]

10. Liberati A, Altman DG, Tetzlaff J, et al. The PRISMA Statement for Reporting Systematic Reviews and Meta-Analyses of Studies that Evaluate Health Care Interventions: Explanation and Elaboration. J Clin Epidemiol. 2009; 62(10):e1-24. [PubMed: 19631507]

11. Lampinen TM, Chan K, Anema A, et al. Self-Screening for Rectal Sexually Transmitted Infections: Human Papillomavirus. Clin Infect Dis. 2006; 42(2):308-309. [PubMed: 16355352]

12. Hernandez BY, McDuffie K, Goodman MT, et al. Comparison of Physician- and Self-Collected Genital Specimens for Detection of Human Papillomavirus in Men. J Clin Micro. 2006; 44(2): 513-517.

13. Lampinen TM, Latulippe L, Van Niekerk D, et al. Illustrated Instructions for Self-Collection of Anorectal Swab Specimens and Their Adequacy for Cytological Examination. Sex Transm Dis. 2006; 33(6):386-388. [PubMed: 16543863]

14. Chin-Hong PV, Berry M, Cheng S, et al. Comparison of Patient- and Clinician-Collected Anal Cytology Samples to Screen for Human Papillomavirus-Associated Anal Intraepithelial Neoplasia in Men Who Have Sex with Men. Ann Intern Med. 2008; 149(5):300-306. [PubMed: 18765699]

15. Wayal S, Llewellyn C, Smith H, et al. Self-Sampling for Oropharyngeal and Rectal Specimens to Screen for Sexually Transmitted Infections: Acceptability Among Men Who Have Sex with Men. Sex Transm Infect. 2009; 85(1):60-64. [PubMed: 18708480]

16. van der Helm JJ, Hoebe CJ, van Rooijen MS, et al. High Performance and Acceptability of SelfCollected Rectal Swabs for Diagnosis of Chlamydia trachomatis and Neisseria gonorrhoeae in Men Who Have Sex with Men and Women. Sex Transm Dis. 2009; 36(8):493-497. [PubMed: 19617869]

17. Dodge B, van der Pol B, Rosenberger JG, et al. Field Collection of Rectal Samples for Sexually Transmitted Infection Diagnostics Among Men Who Have Sex with Men. Int J STD AIDS. 2010; 21(4):260-264. [PubMed: 20378897]

18. Gilbert M, Kwag M, Mei W, et al. Feasibility of Incorporating Self-Collected Rectal Swabs Into a Community Venue-Based Survey to Measure the Prevalence of HPV Infection in Men Who Have Sex with Men. Sex Transm Dis. 2011; 38(10):964-969. [PubMed: 21934574]

19. Soni S, White JA. Self-Screening for Neisseria gonorrhoeae and Chlamydia trachomatis in the Human Immunodeficiency Virus Clinic-High Yields and High Acceptability. Sex Transm Dis. 2011; 38(12):1107-1109. [PubMed: 22082720]

20. Read TR, Hocking JS, Vodstrcil LA, et al. Oral Human Papillomavirus in Men Having Sex with Men: Risk-Factors and Sampling. PLoS One (Online). Nov.2012 7(11):e49324. [Accessed April 20, 2016] Available from: http://journals.plos.org/plosone/.

21. Cherneskey MA, Jang D, Portillo E, et al. Self-Collected Swabs of the Urinary Meatus Diagnose More Chlamydia trachomatis and Neisseria gonorrhoeae Infections Than First Catch Urine from Men. Sex Transm Infect. 2013; 89(2):102-104. [PubMed: 23024224] 
22. Chai SJ, Aumakhan B, Barnes M, et al. Internet-Based Screening for Sexually Transmitted Infections to Reach Nonclinic Populations in the Community: Risk Factors for Infection in Men. Sex Transm Dis. 2010; 37(12):756-763. [PubMed: 20644498]

23. Dize L, Agreda P, Quinn N, et al. Comparison of Self-Obtained Penile-Meatal Swabs to Urine for the Detection of C. trachomatis, N. gonorrhoeae and T. vaginalis. Sex Transm Infect. 2013; 89(4): 305-307. [PubMed: 23093735]

24. Hernandez BY, Wilkens LR, Unger ER, et al. Evaluation of Genital Self-Sampling Methods for HPV Detection in Males. J Clin Virol. 2013; 58(1):168-175. [PubMed: 23880161]

25. Gaydos CA, Barnes MR, Quinn N, et al. Trichomonas vaginalis Infection in Men Who Submit Self-Collected Penile Swabs After Internet Recruitment. Sex Transm Infect. 2013; 89(6):504-508. [PubMed: 23354525]

26. Keaveney S, Sadlier C, O'Dea S, et al. High Prevalence of Asymptomatic Sexually Transmitted Infections in HIV-Infected Men Who Have Sex with Men: A Stimulus to Improve Screening. Int J STD AIDS. 2014; 25(10):758-761. [PubMed: 24480850]

27. Wood M, Ellks R, Grobicki M. Outreach Sexual Infection Screening and Postal Tests in Men Who Have Sex with Men: Are They Comparable to Clinic Screening? Int J STD AIDS. 2015; 26(6): 428-431. [PubMed: 24912535]

28. Dize L, Barnes P, Barnes M, et al. Performance of Self-Collected Penile-Meatal Swabs Compared to Clinician-Collected Urethral Swabs for the Detection of Chlamydia trachomatis, Neisseria gonorrhoeae, Trichomonas vaginalis, and Mycoplasma genitalium by Nucleic Acid Amplification Assays. Diagn Microbiol Infect Dis. 2016; 86(2):131-135. [PubMed: 27497595]

29. Nyatsanza F, Trivedy A, Brook G. The Effect of Introducing Routine Self-Taken Extra-Genital Swabs in a Genitourinary Medicine Clinic Cohort: A Before and After Study. Int J STD AIDS. 2016; 27(14):1330-1333. [PubMed: 26672002]

30. Chernesky M, Jang D, Smieja M, et al. Urinary Meatal Swabbing Detects More Men Infected with Mycoplasma genitalium and Four Other Sexually Transmitted Infections Than First Catch Urine. Sex Transm Dis. 2017; 44(8):489-491. [PubMed: 28703728]

31. Centers for Disease Control and Prevention. [Accessed September 28, 2017] 2015 Sexually Transmitted Diseases Treament Guidelines: Emerging Issues (CDC Web site). Available at: https:// www.cdc.gov/std/tg2015/emerging.htm\#myco 


\section{SUMMARY}

A systematic review evaluating the validity, feasibility, and acceptability of using selfcollected swabs for detection of sexually transmitted infections in adult men. 
STI Sample Self-Collection in Men - PRISMA Flow Diagram

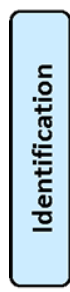

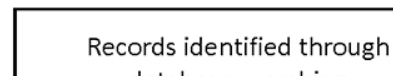
database searching $(n=1050)$
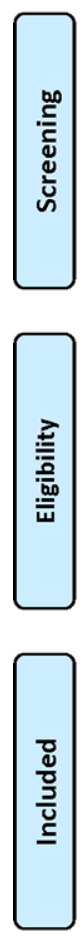

Additional records identified through other sources $(n=3)$
Studies included in qualitative synthesis $(n=20)$

Records excluded ( $n=501$ )

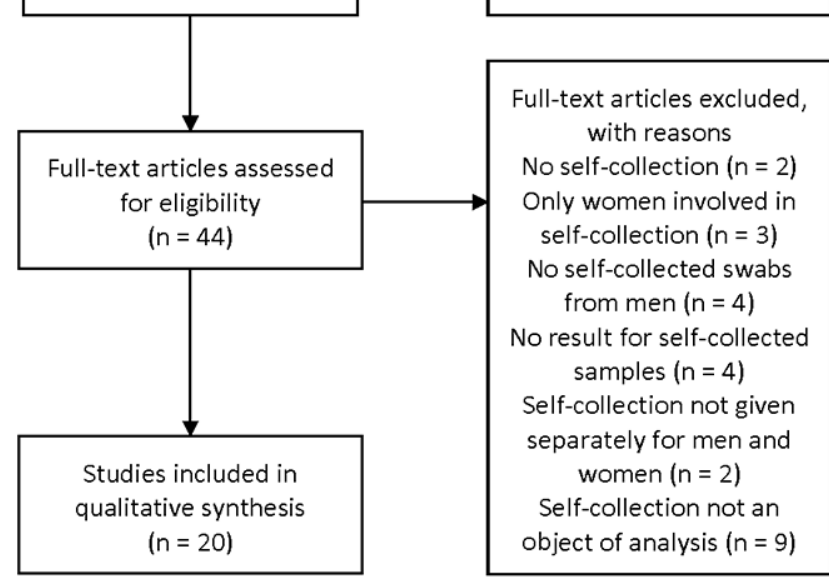

From: Moher D, Liberati A, Tetzlaff J, Altman DG, The PRISMA Group (2009). Preferred Reporting ftems for Systematic Reviews and MetaAnalyses: The PRISMA Statement. PLoS Med 6(7): e1000097. doi:10.1371/journal.pmed1000097

For more information, visit www.prisma-statement.org.

Figure 1.

PRISMA flow diagram showing screening process (adapted). ${ }^{8}$ 

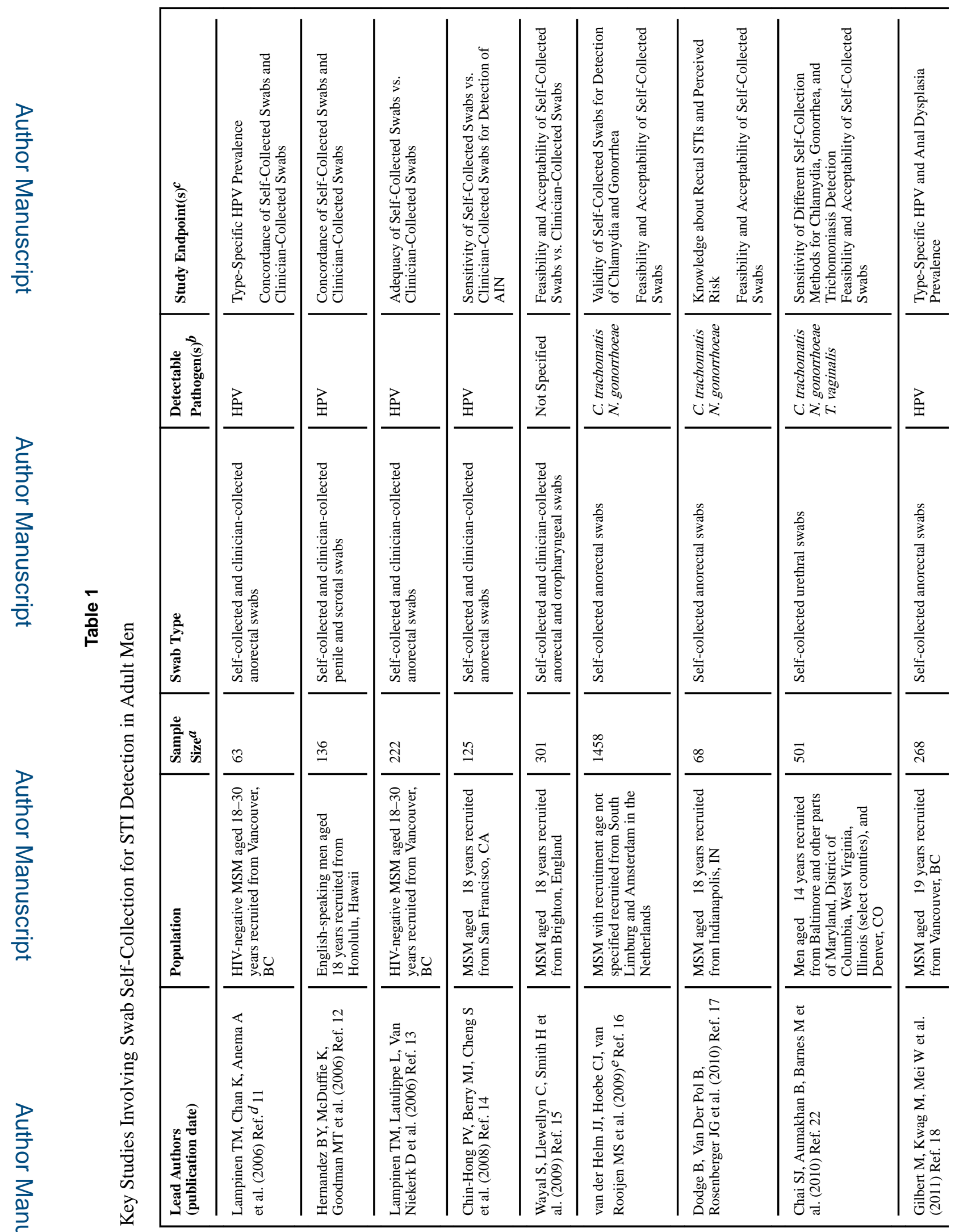

Sex Transm Dis. Author manuscript; available in PMC 2019 May 01. 


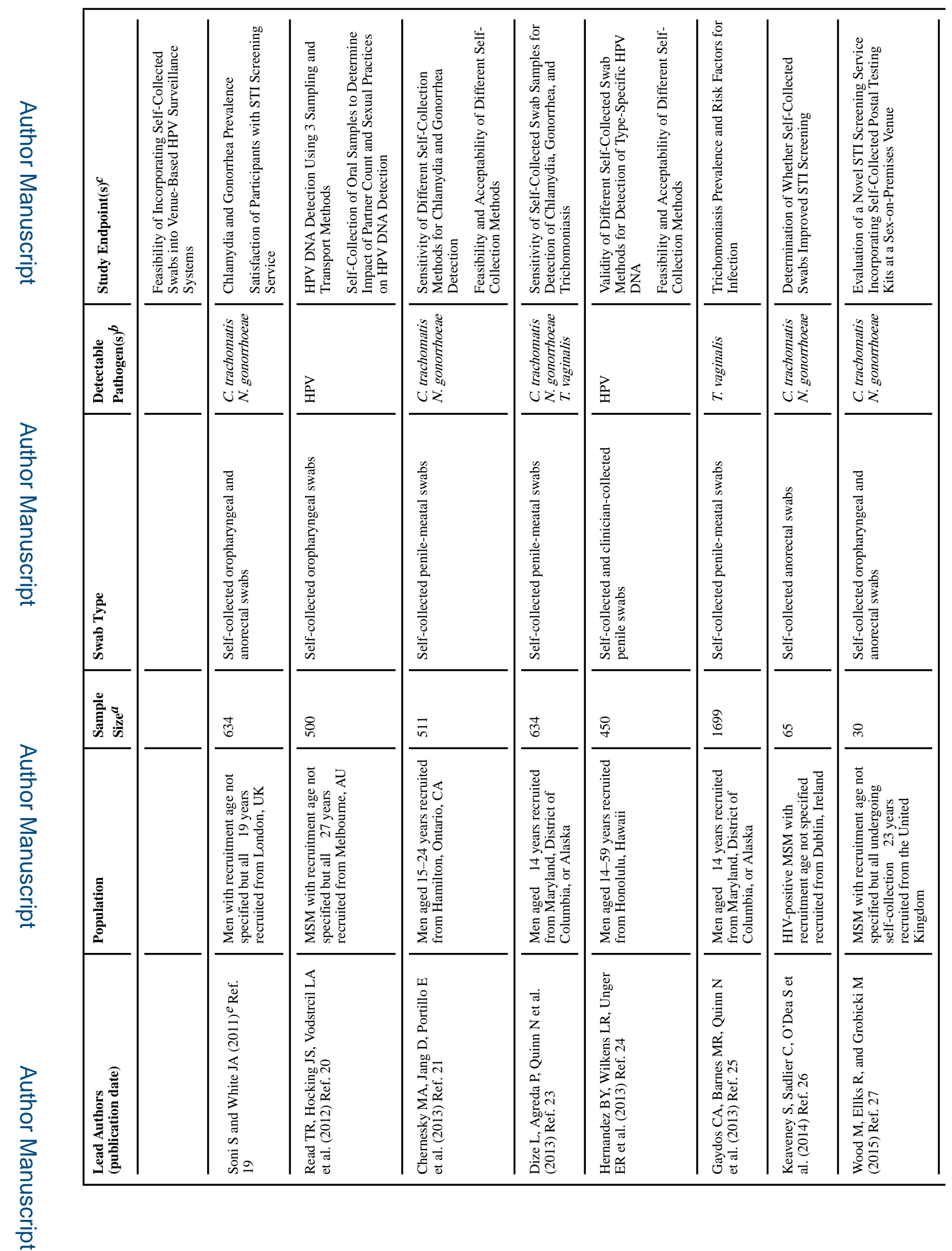

Sex Transm Dis. Author manuscript; available in PMC 2019 May 01. 


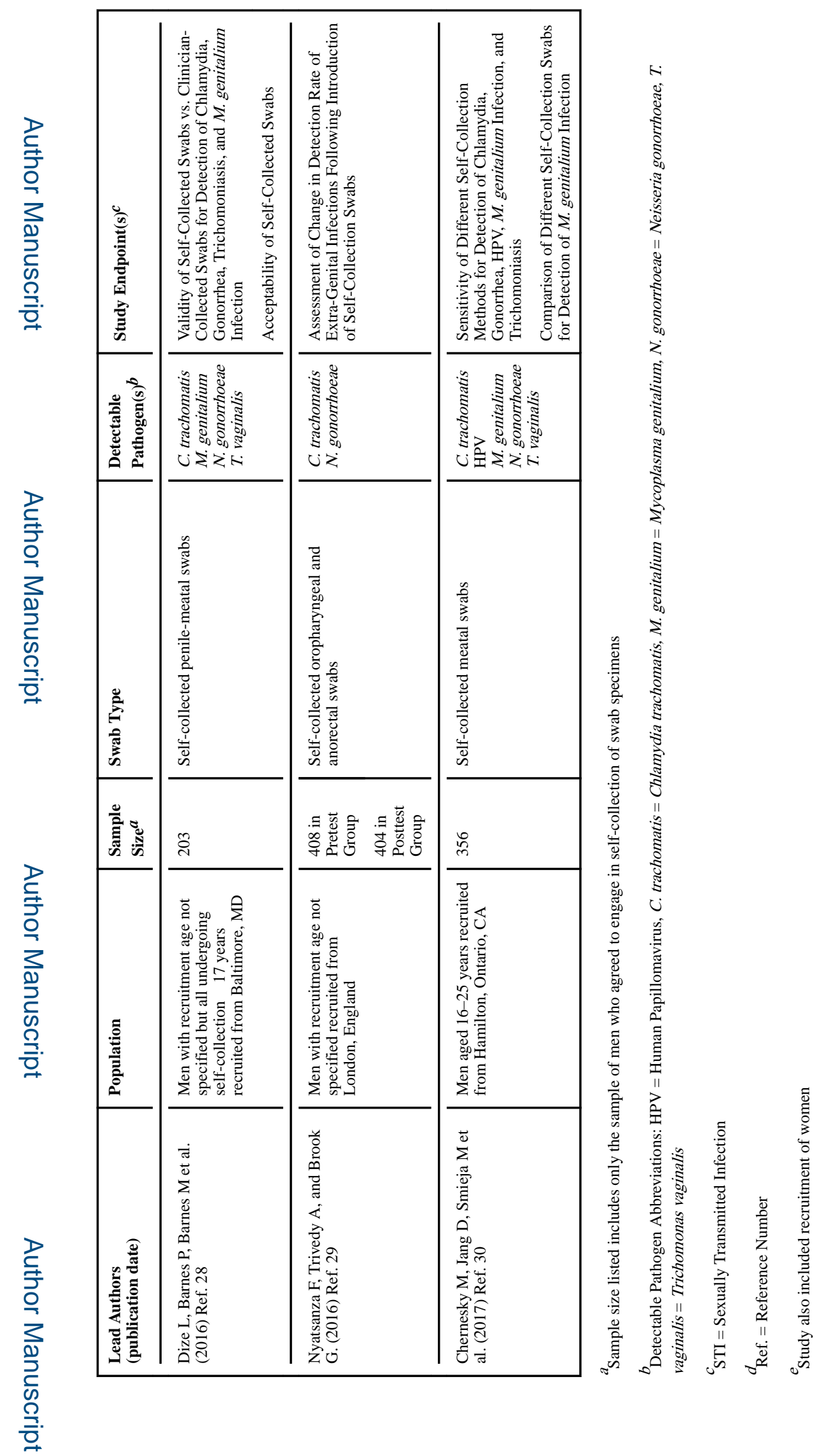

Sex Transm Dis. Author manuscript; available in PMC 2019 May 01. 
Table 2

Matched Self-Collected Swabs vs. Clinician-Collected Swabs for Detection of Human Papillomavirus (HPV) Infection

\begin{tabular}{lllll} 
Study & Swab Type & Collection Method & Measure & Result (\%) \\
\hline Lampinen TM, Chan K et al. (2006) & Anorectal Swab & Self-Collection & HR-HPV Prevalence & 67 \\
& & Clinician-Collection & HR-HPV Prevalence \\
Hernandez BY, McDuffie K et al. (2006) & Genital Swab & Self-Collection & HPV Prevalence & 42.8 \\
Hernandez BY, Wilkens Lynne R et al. (2013) & Genital Swab & Self-Collection & HPV Prevalence & 41.3 \\
& & Clinician-Collection & HPV Prevalence & 47.9 \\
\hline
\end{tabular}

$\hat{H R}-\mathrm{HPV}=$ High-risk human papillomavirus types 